\title{
Urinary peptidomics reveals proteases involved in idiopathic membranous nephropathy
}

\author{
Baoxu Lin, Jianhua Liu, Yue Zhang, Yabin Wu, Shixiao Chen, Yibo Bai, Qiuying Liu and Xiaosong Qin*
}

\begin{abstract}
Background: Idiopathic membranous nephropathy (IMN) is a cause of nephrotic syndrome that is increasing in incidence but has unclear pathogenesis. Urinary peptidomics is a promising technology for elucidating molecular mechanisms underlying diseases. Dysregulation of the proteolytic system is implicated in various diseases. Here, we aimed to conduct urinary peptidomics to identify IMN-related proteases.

Results: Peptide fingerprints indicated differences in naturally produced urinary peptide components among 20 healthy individuals, 22 patients with IMN, and 15 patients with other kidney diseases. In total, 1,080 peptidematched proteins were identified, 279 proteins differentially expressed in the urine of IMN patients were screened, and 32 proteases were predicted; 55 of the matched proteins were also differentially expressed in the kidney tissues of IMN patients, and these were mainly involved in the regulation of proteasome-, lysosome-, and actin cytoskeleton-related signaling pathways. The 32 predicted proteases showed abnormal expression in the glomeruli of IMN patients based on Gene Expression Omnibus databases. Western blot revealed abnormal expression of calpain, matrix metalloproteinase 14, and cathepsin S in kidney tissues of patients with IMN.
\end{abstract}

Conclusions: This work shown the calpain/matrix metalloproteinase/cathepsin axis might be dysregulated in IMN. Our study is the first to systematically explore the role of proteases in IMN by urinary peptidomics, which are expected to facilitate discovery of better biomarkers for IMN.

Keywords: Urinary peptidomics, Idiopathic membranous nephropathy, Proteases

\section{Background}

Idiopathic membranous nephropathy (IMN), a known cause of nephrotic syndrome, is a public health concern estimated to affect $1-18$ people in a population of 100,000 globally [1]. Proteinuria has been reported in $75-85 \%$ of patients with IMN, and $40-60 \%$ of IMN patients may progress to end-stage renal failure within 520 years [1]. Thus, urinary protein level is an important indication for diagnosing, planning the treatment, and evaluating the progress of IMN [2].

\section{*Correspondence: qinxs@sj-hospital.org}

Department of Laboratory Medicine, Shengjing Hospital of China Medical University, No. 36 Sanhao Street, Heping District, 110004 Shenyang, P. R. China
Proteinuria and related symptoms are considered clinical manifestations of podocyte injury in IMN [3]. The involvement of kidney injury in IMN was identified by pathological examination of kidney tissue using immunofluorescence and electron microscopy in the $1940 \mathrm{~s}$ [4]. However, the molecular mechanism underlying kidney injury remains unclear. In 2009, an antiphospholipase A2 receptor (anti-PLA2R) antibody was discovered in the sera of IMN patients [1]. Since then, it has been used in the diagnosis and therapeutic targeting of IMN, with about $70-80 \%$ of patients being positive for this marker [5]. Nevertheless, the mechanism underlying anti-PLA2R antibody production in these patients has not been fully clarified. Therefore, in this context,

(c) The Author(s). 2021 Open Access This article is licensed under a Creative Commons Attribution 4.0 International License, which permits use, sharing, adaptation, distribution and reproduction in any medium or format, as long as you give appropriate credit to the original author(s) and the source, provide a link to the Creative Commons licence, and indicate if changes were made. The images or other third party material in this article are included in the article's Creative Commons licence, unless indicated otherwise in a credit line to the material. If material is not included in the article's Creative Commons licence and your intended use is not permitted by statutory regulation or exceeds the permitted use, you will need to obtain permission directly from the copyright holder. To view a copy of this licence, visit http://creativecommons.org/licenses/by/4.0/. The Creative Commons Public Domain Dedication waiver (http://creativecommons.org/publicdomain/zero/1.0/) applies to the data made available in this article, unless otherwise stated in a credit line to the data. 
the main goal is to unravel the pathogenic molecular mechanism underlying kidney damage.

A complex protease network is at play in podocytes [6-8], and protease-dependent signaling pathways are reportedly related to inflammation and tissue remodeling in kidney diseases [9]. Therefore, proteases are becoming increasingly important in targeted therapy of renal diseases [3].

Large-scale research on proteins is termed proteomics, whereas that on naturally occurring peptides is termed peptidomics $[9,10]$. High-resolution mass spectrometry (MS), especially data-independent acquisition MS (DIAMS), has enabled broad application of proteomics in medical science. Urinary peptidomics is a promising technology to elucidate the pathogenesis of IMN [11], because the urine may contain low-molecular-weight peptides produced by endogenous protease activity in the kidneys $[12,13]$, and the action mechanism of proteases in kidney diseases, which has been mainly reported in diabetic nephropathy [14, 15].

A typical example of application of urinary peptidomics is development of urinary peptide biomarkers, such as the CKD 273 classifier that has been proposed for diagnosis of chronic kidney disease and is undergoing clinical trials [16]. Recent studies explored the mechanisms underlying urinary peptide production in gastric cancer [17] and Parkinson's disease [18], further indicating that urine reflects changes in biological pathways at the tissue level.

In this study, we aimed to elucidate the biological processes and signaling pathways involved in IMN-related protein dysregulation and tissue injury by analyzing naturally produced urinary peptides differentially expressed in IMN patients, identifying proteins matched to these peptides, and predicting and validating the proteases involved in the production of these peptides. Our findings are expected to serve as a reference for studies on the pathological mechanism underlying IMN and discovery of related biomarkers.

\section{Results}

\section{Clinical characteristics of subjects}

Clinical information of subjects is summarized in Table 1. The estimated glomerular filtration rate (eGFR) was calculated with CKD-EPI (Chronic Kidney Disease Epidemiology Collaboration) Cystatin C formula (2012). eGFR of IMN and other nephropathy groups was lower than that of the HC group.

\section{Global characteristics of urinary peptidomics of IMN}

The workflow of the study is shown in Fig. 1. Peptide fingerprint of each sample for all groups was profiled using MALDI-TOF MS; one sample representative of each group is shown in Fig. 2, which shows the
Table 1 Comparison of demography and eGFR between IMN, $\mathrm{OD}$, and $\mathrm{HC}$ groups

\begin{tabular}{|c|c|c|c|c|}
\hline & $\mathrm{HC}$ & IMN & OD & $P$ \\
\hline Sex (Male/Female) ${ }^{a}$ & $10 / 10$ & $13 / 9$ & $8 / 7$ & $>0.05$ \\
\hline $\begin{array}{l}\text { Age } \\
(\text { mean } \pm S D)^{b}\end{array}$ & $47.2 \pm 11.9$ & $50.2 \pm 10.7$ & $42.0 \pm 15.0$ & 0.84 \\
\hline $\begin{array}{l}\mathrm{eGFR} / \mathrm{mL} / \mathrm{min} / 1.73 \mathrm{~m}^{2} \\
(\mathrm{mean} \pm \mathrm{SD})^{\mathrm{b}^{*}}\end{array}$ & $113.8 \pm 19.4$ & $79.8 \pm 22.1$ & $53.7 \pm 25.9$ & $<0.01$ \\
\hline
\end{tabular}

${ }^{*}$ Indicates there is significant difference among $\mathrm{HC}$, IMN and OD groups, $P$ $<0.05$;

${ }^{a}$ and ${ }^{b}$ indicate that Chi-Square test and one-way analysis of variance were applied, respectively

eGFR, estimated glomerular filtration rate; IMN, idiopathic membranous nephropathy; OD, other kidney disease; $\mathrm{HC}$, healthy control; SD,

standard deviation

differences in the urinary peptide fingerprints among healthy individuals and patients with kidney disease. Altogether, these results proved that we successfully extracted meaningful peptides from the urine samples.

In DIA-MS, 1080 peptide-matched proteins were identified, 559 proteins were quantified. In a qualitative analysis of differentially expressed proteins (DEPs) in IMN, 248 proteins were identified as unique to IMN patients. In quantitative analysis, 316 proteins were identified in $\mathrm{HC}$, IMN, and OD groups. The comparison of the IMN and $\mathrm{HC}$ groups $(\mathrm{IMN} / \mathrm{HC}$ ) revealed 125 (fold change > 1.5 ) and 121 (fold change < 1.5) upregulated and downregulated proteins, respectively, whereas the comparison between the $\mathrm{OD}$ and $\mathrm{HC}$ groups $(\mathrm{OD} / \mathrm{HC})$, identified 127 (fold change $>1.5$ ) and 120 (fold change $<1.5$ ) upregulated and downregulated proteins, respectively. The shared upregulated and downregulated proteins in the two comparisons were 18 and 13, respectively. Thus, 279 IMN DEPs were selected (Fig. 1).

\section{Kidney-originated urinary proteins and GO enrichment}

Among 279 IMN DEPs, 158 kidney-originated urinary proteins were predicted based on sc-RNA seq database analysis (Fig. 3 A). Protein expression patterns indicated that most proteins might be from podocytes.

ClueGO software was used to analyze the interaction network of biological processes of 158 DEPs and revealed that these proteins were related to 60 biological processes, mainly divided into four categories (Fig. $4 \mathrm{~A}$ ): (1) signaling pathways related to cellular pathological changes; (2) biological processes related to cell metabolism and cell activity; (3) biological processes related to intracellular protein transport; and (4) others. Among these, biological processes include oxidative stress and oxidative stress-induced apoptosis signaling pathway and interleukin-12-mediated signaling pathway. The second type comprises antigen processing and presentation, and amino acid metabolism. The third type contains the directional transport of intracellular proteins to the cell membrane and endoplasmic reticulum. The fourth type 


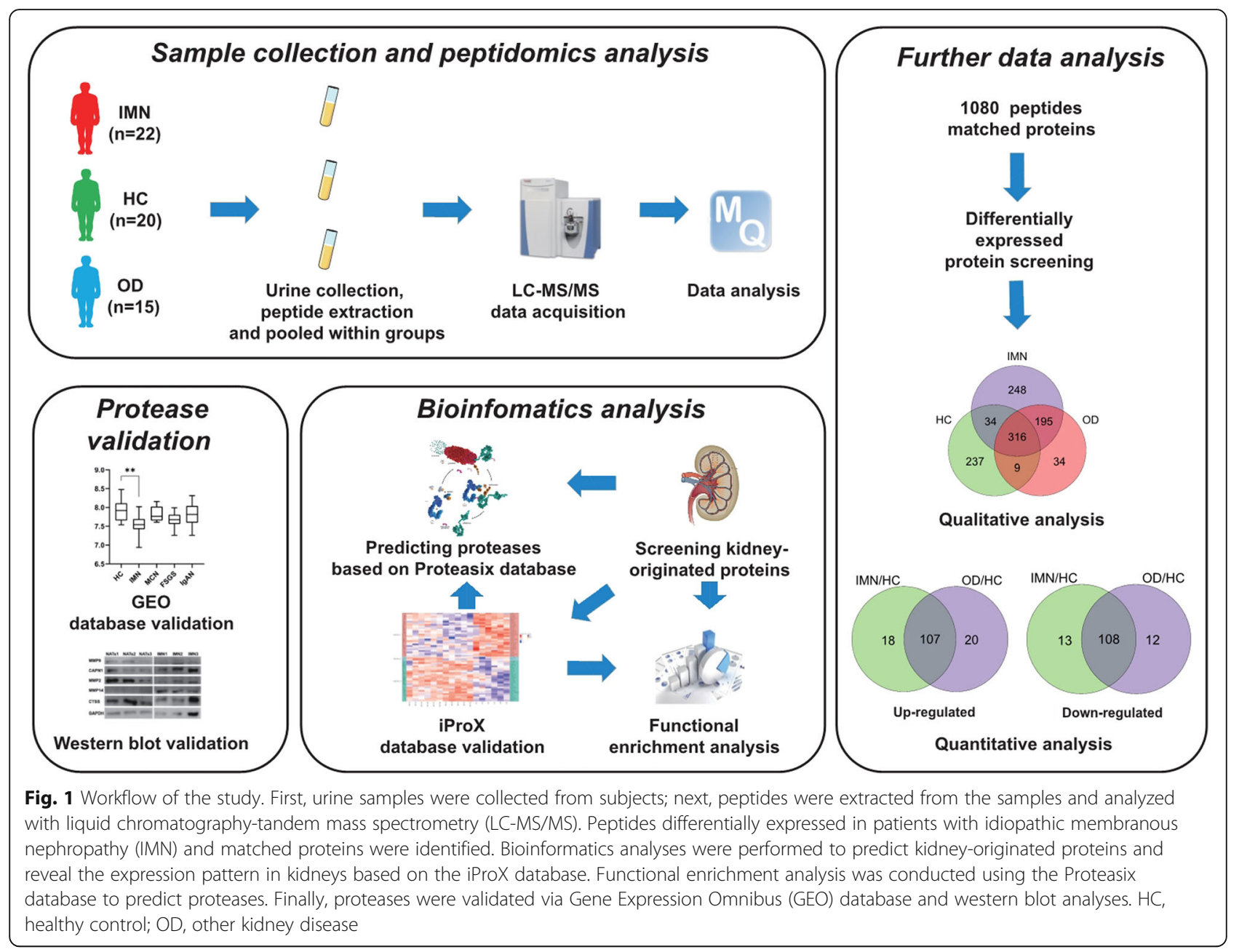

included cytoskeleton composition and membrane lipid metabolism.

The cellular localization of 158 DEPs and the involved signaling pathways were analyzed using DAVID. The results showed that the cellular localization of these proteins mainly included cell surface, cytoplasm, intracellular organelles, intracellular organelle lumen, intimal system, extracellular region, cell vesicle, lysosome, proteasome complex, etc. (Fig. 4B). The signaling pathways involved by these proteins included proteasome, lysosome, regulation of actin cytoskeleton, antigen processing and presentation, ribosome and metabolic pathway, etc. (Fig. 4B). These results indicate that the IMN DEPs may reflect the pathological changes in various metabolic processes.

\section{Validation of IMN DEPs in kidney tissue}

To explore the expression of urinary peptide-matched proteins in the kidney, we investigated 158 urinary IMN DEPs using the iProX proteomics database. We obtained the quantitative analysis data of 131 urinary DEPs; 55 DEPs were identified between HC and IMN groups, of which 28 were upregulated and 27 were downregulated in IMN patients (Fig. 3B).

GO enrichment analysis showed that both upregulated and downregulated proteins were related to protein degradation. The biological processes related to the upregulated proteins included proteolysis and proteasomemediated protein catabolism. The biological processes related to the downregulated proteins included cell adhesion and protein binding (Fig. 5B). The cellular localization of upregulated proteins was in the proteasome and lysosome. Downregulated proteins were located in the extracellular matrix and cytoskeleton (Fig. 5 A). Upregulated proteins participated in lysosomal and proteasome signaling pathways, whereas downregulated proteins were involved in metabolic signaling pathways (Fig. $5 \mathrm{C}$ ). These results suggested that some damaging changes may occur in the kidneys of IMN patients, such as enhanced proteolysis and decreased intercellular adhesion. The peptides associated with these proteins may be released into the urine; thus, the level of peptides in the urine may reflect the severity of kidney tissue injury. 


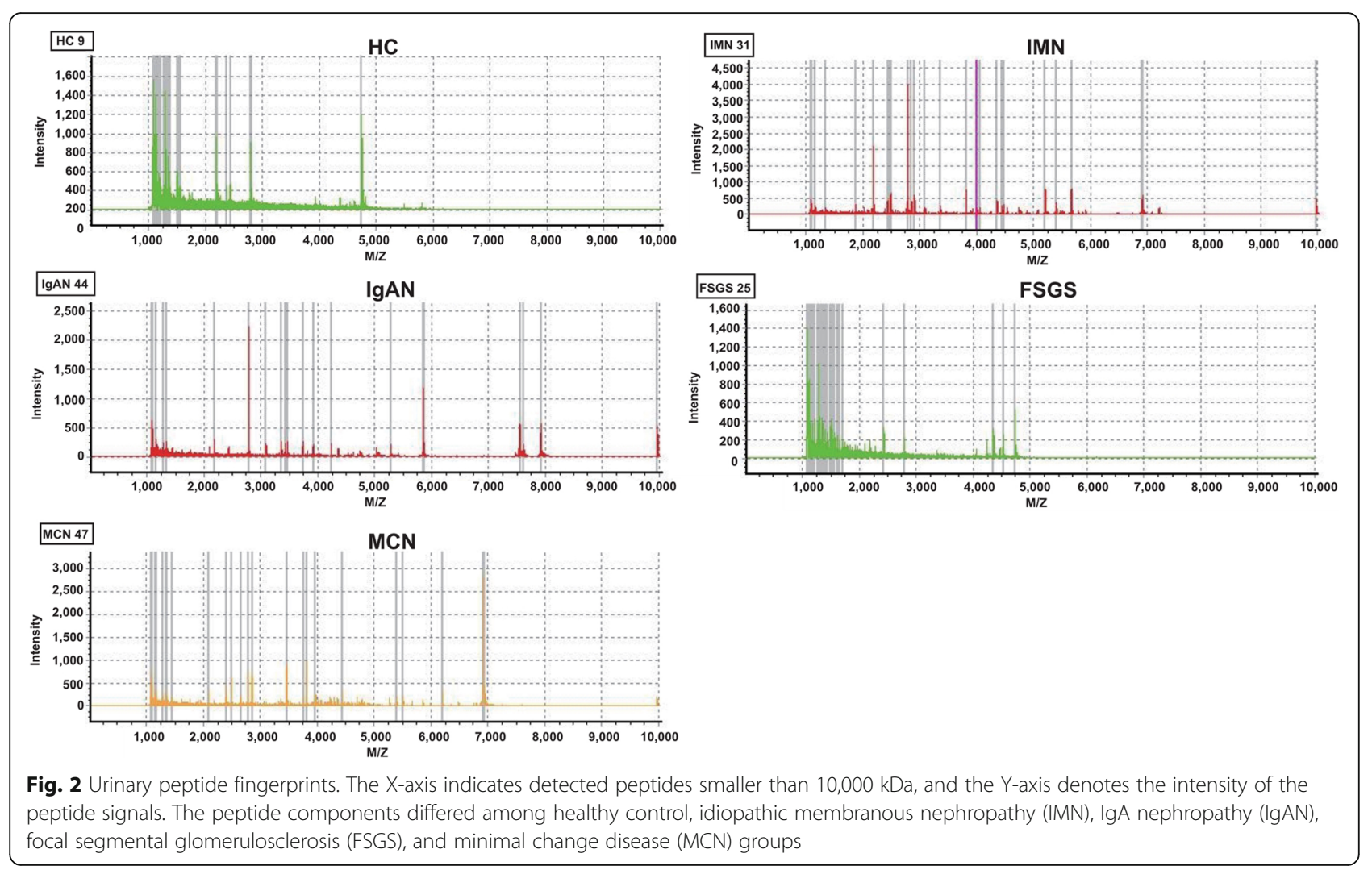

\section{Protease prediction and validation in the glomerulus}

To link urinary peptides with proteases, Proteasix database analysis was used to predict proteases that may cleave the 158 proteins identified in this study. In total, 32 proteases were predicted (Table 2).

Dysregulation of proteases in glomeruli may be the cause underlying the production of urinary peptides. Therefore, we explored the expression patterns of predicted proteases in the GSE99339 dataset based on GEO. This dataset contains mRNA expression data of 27 protease genes related to different kidney diseases. Compared with the HC group, the IMN group showed lower expression of CAPN1, GZMK, and MMP14 $(P<$ $0.05)$ and higher expression of CAPN2 and DAG1 $(P<$ 0.05). Although CTSS expression was higher in the IMN group, the difference was not statistically significant (Fig. $6 \mathrm{~A}$ ). This result suggested that the glomerular protease spectrum of IMN is different from that of $\mathrm{HC}$, leading to pathological changes in glomerular function.

\section{Validation of proteases in IMN kidney tissue}

Different proteases, such as calpain, cathepsin, and matrix metalloproteinases, are reportedly dysregulated in CKD [19-21]. The gelatinases, MMP2 and MMP9, mediate
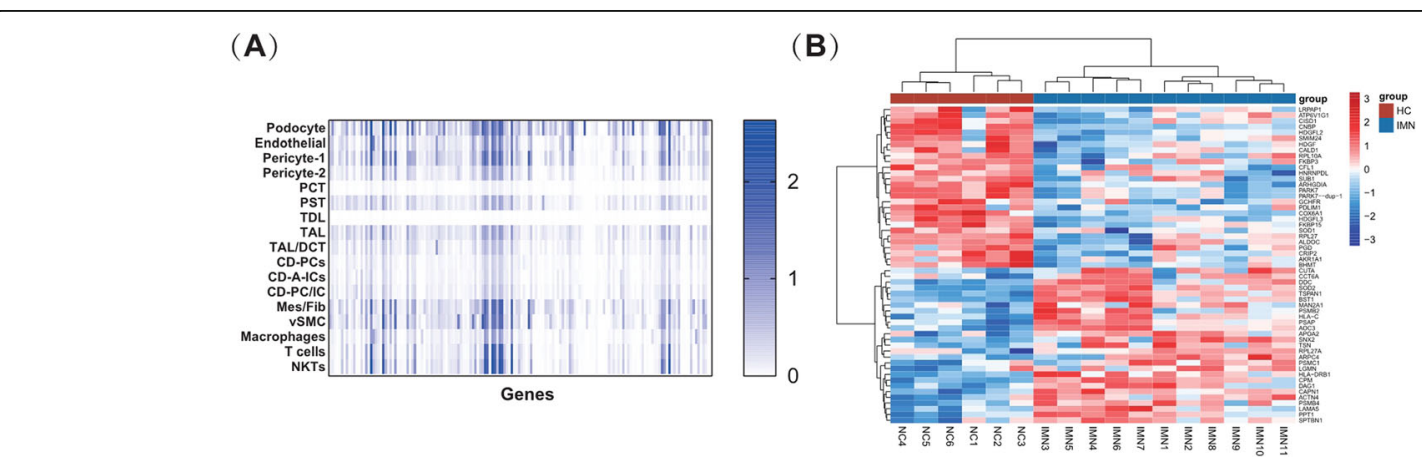

Fig. 3 Heat maps showing (A) the expression pattern of genes encoding the 158 kidney-originated proteins in single renal cells and (B) 55 differentially expressed proteins (DEP) in kidney tissue from patients with idiopathic membranous nephropathy (IMN) based on iProX database analysis 


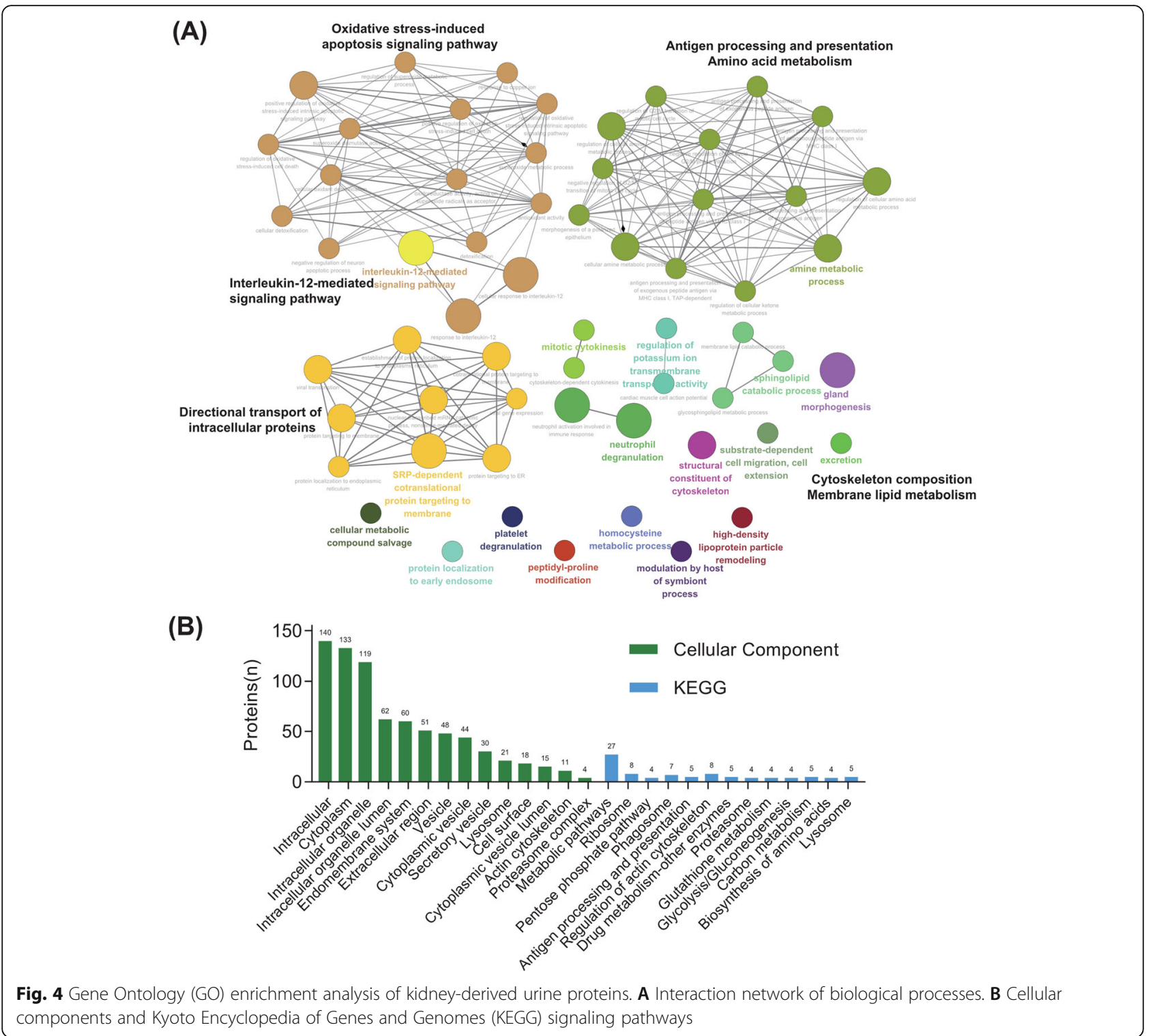

fibrosis in CKD, such as in diabetes mellitus [21]. Therefore, the expression of CAPN1, MMP2, MMP9, MMP14, and CTSS in kidney tissues of 3 IMN and 3 NATs were detected using western blotting (Fig. 6B). Compared with NATs, the IMN kidney tissue showed higher expression of CAPN1, MMP14, and CTSS; however, this difference was not statistically significant $(P>0.05)$ (Fig. 6 C). MMP2 and MMP9 levels also showed no apparent differences between the two groups (Fig. 6 C). These results suggest that specific proteases are dysregulated in kidney tissues of patients with IMN. Nevertheless, further validation of this protease dysregulation is warranted.

\section{Discussion}

In this study, we examined urinary peptides and identified peptide-matched proteins to reveal the protein dysregulation related to IMN. To achieve highthroughput screening and save costs incurred by MS, we adopted the mixed sample strategy. Some studies have used capillary electrophoresis-MS or MALDI-TOF MS to analyze the peptides in human samples and identified the amino acid sequences. However, they also matched the identified peptide with corresponding proteins and studied the functions of the proteins [22, 23]. Here, we aimed to elucidate the production of urinary peptides and related proteases. Therefore, enriched urinary peptides were analyzed using routine proteomic methods, and matched proteins were identified easily.

Our results showed that 248 peptide-matched proteins were unique to IMN. It is generally believed that under the "label-free" quantitative strategy, there will be a 10 $50 \%$ loss of quantitative information of peptides and 


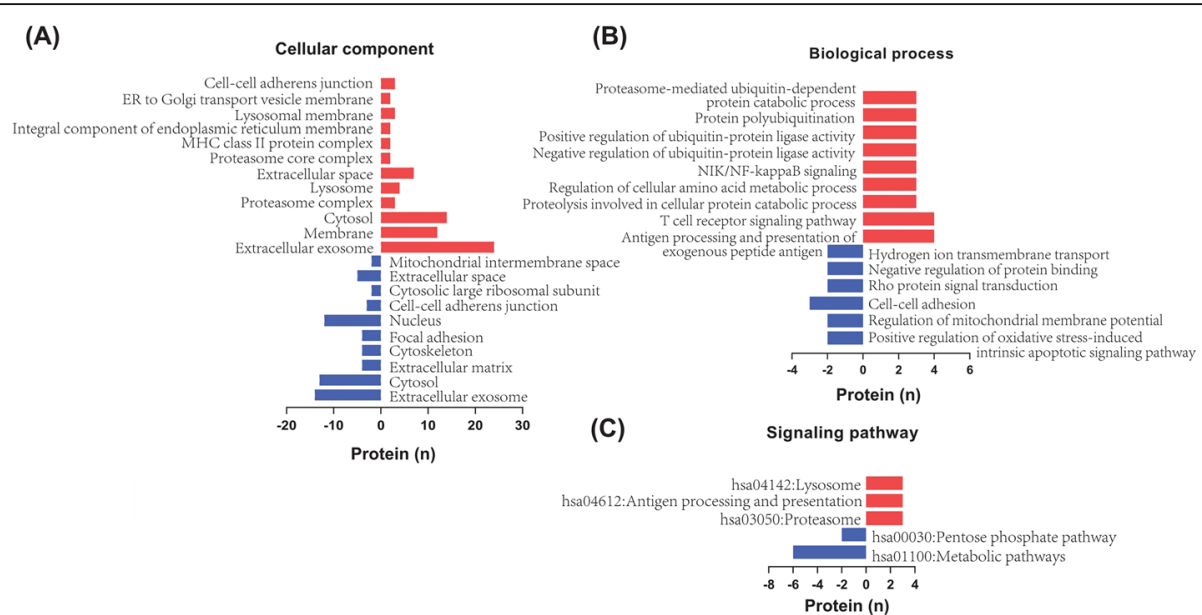

Fig. 5 Functional annotation of differentially expressed proteins validated in iProX. A Cellular components; (B) Biological processes; (C) Signaling pathways. Upregulated and downregulated pathways are indicated by red (right) and blue (left) bars

proteins, possibly because the content of some peptides is below the detection limit of the MS [24]. Therefore, it can be considered that the peptides were identified due to their high abundance. According to quantitative analysis, 31 proteins were dysregulated specifically in IMN. Thus, this study shows differences in urinary peptides among IMN patients, healthy individuals, and individuals with other kidney diseases, consistent with previous reports stating that the urine proteome can distinguish distinct subtypes of different disorders and disease conditions [25].

Studies have been conducted on urine proteomics of IMN [26, 27]. Ngai et al. [26] found that the function of 37 IMN urinary DEPs was involved in the signaling pathways related to the regulation of renal filtration. Pang $\mathrm{Lu}$ et al. [27] showed that the screened IMN urinary DEPs were associated with complement activation and coagulation cascade reactions. Besides the proteins mentioned above, the IMN urinary DEPs selected in this study were also involved in regulating proteasome, lysosome, actin cytoskeleton, antigen processing and presentation, and other signaling pathways, which may cause the production of endogenous peptides. However, the research on understanding the role of proteases and peptidomics in IMN is still very limited.

Table 2 List of 32 proteases predicted using the Proteasix tool

\begin{tabular}{llll}
\hline Protein symbol & Protein name & Protein symbol & Protein name \\
\hline CAPN1 & calpain 1 & GZMB & granzyme B \\
CAPN2 & calpain 2 & GZMK & granzyme K \\
CAPN3 & calpain 3 & GZMM & granzyme M \\
CASP1 & caspase 1 & HPN & hepsin \\
CASP2 & caspase 2 & LGMN & legumain \\
CASP3 & caspase 3 & MEP1A & meprin A subunit alpha \\
CASP6 & caspase 6 & MEP1B & meprin A subunit beta \\
CASP9 & caspase 9 & MMP1 & matrix metallopeptidase 1 \\
CTSB & cathepsin B & MMP14 & matrix metallopeptidase 14 \\
CTSG & cathepsin G & MMP2 & matrix metallopeptidase 2 \\
CTSK & cathepsin K & MMP3 & matrix metallopeptidase 3 \\
CTSL & cathepsin L & MMP7 & matrix metallopeptidase 7 \\
CTSS & cathepsin S & ST14 & suppression of tumorigenicity 14 \\
DAG1 & dystroglycan 1 & TMPRSS11E & transmembrane protease, serine 11E \\
ELANE & elastase, neutrophil expressed & TMPRSS6 & transmembrane protease, serine 6 \\
GZMA & granzyme A & TMPRSS7 & transmembrane protease, serine 7 \\
\hline
\end{tabular}




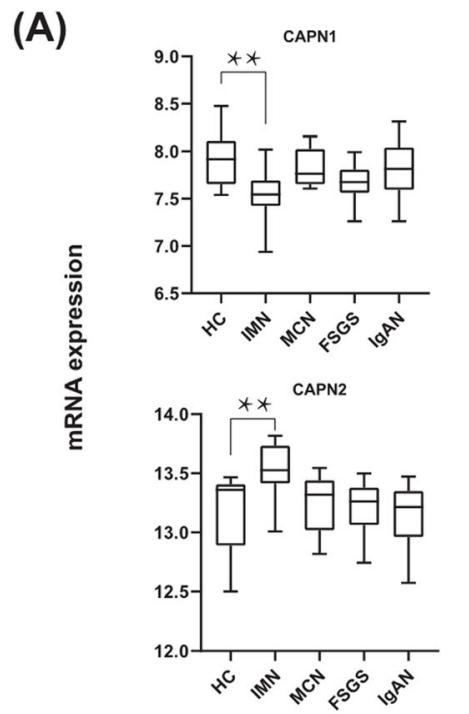

(B)

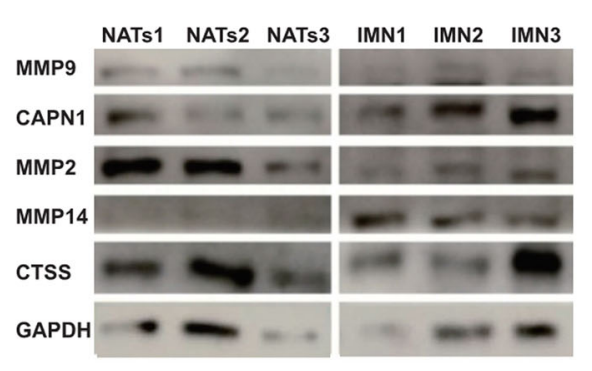

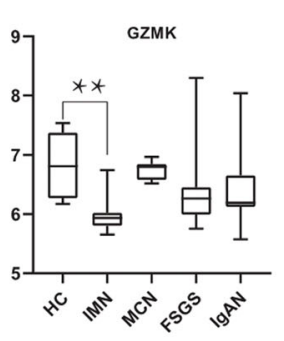
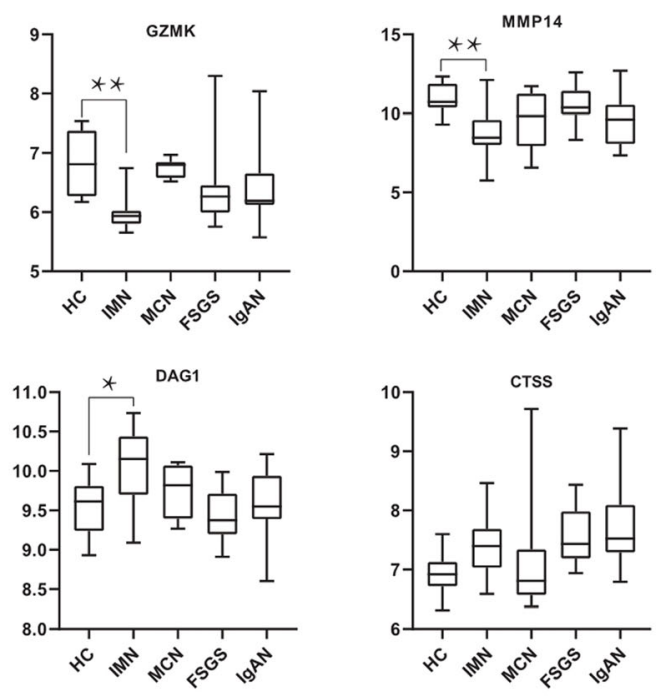

(C)

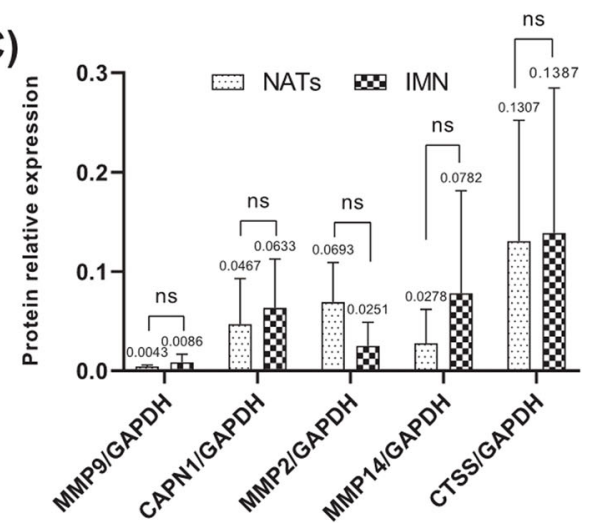

Fig. 6 Protease validation using Gene Expression Omnibus (GEO) database and western blot analyses. A Proteases differentially expressed in idiopathic membranous nephropathy (IMN). B and (C) Western blot showing relative expression of proteases. ${ }^{*} P<0.05 ;{ }^{* *} P<0.01$; ns: $P>0.05$

Proteases are necessary to maintain tissue homeostasis, and their dysregulation may be related to the production of urinary peptides in CKD $[3,9,10]$. Proteasix, a bioinformatics tool, is dedicated to exploring proteases that produce peptides. Brondani et al. [28] and Krochmal et al. [15] predicted various proteases related to the production of urinary peptides in diabetes using Proteasix. In this study, 32 proteases involved in IMN urinary peptide production were predicted by Proteasix. Further validation via western blotting indicated that the levels of proteases CAPN1, MMP14, and CTSS were higher in kidney tissue of IMN, whereas those of the proteases MMP2 and MMP9 did not change significantly. This was not completely consistent with the expression patterns of predicted protease in the GEO dataset, indicating that gene and protein expression trends might not be completely consistent [29]. This study proved that proteases uniquely dysregulated in IMN may be involved with urinary peptide production and that several proteases may be the driving factors of disease progression, which should be studied further.

There are mainly three intracellular proteolytic systems in the body: calpain, lysosome, and ubiquitinproteasome system [30]. Calpains are a group of neutral cysteine proteases that are specifically activated by calcium ions. Calpain family members not only participate in protein hydrolysis and cytoskeleton remodeling but also cell cycle regulation and apoptosis [31, 32]. The balance of protein metabolism maintained by the calpain system will be disrupted by increased $\mathrm{Ca}^{2+}$ concentration. The abnormal or maladjusted activation of calpain is related to some pathological conditions [32].

Transient receptor potential cation channel 6 (TRPC6), a member of transient receptor potential cation channel protein superfamily [33], expressed in glomerular podocytes [40], is the most important ion channel mediating calcium influx in podocytes [33].

In addition, TRPC6, nephrin, and podocin constitute the podocyte SD complex, which maintains the normal 
function of the glomerulus, and its dysregulation causes proteinuria and glomerular diseases [33, 34]. Reiser et al. found that the expression level of TRPC6 mRNA in IMN patients was significantly higher than that in normal individuals [35]. Furthermore, the bound anti$\mathrm{PLA}_{2} \mathrm{R}$ antibody and target antigen may activate $\mathrm{Ca}^{2+}$ influx and/or promote TRPC6 expression [35].

Therefore, elevated intracellular $\mathrm{Ca}^{2+}$ levels enhance calpain activity, which may destroy podocytes and other cells. Studies have shown that increased intracellular $\mathrm{Ca}^{2+}$ levels activate calpain, which can hydrolyze cytoskeletal proteins actin [36], cleave apoptosis-related proteins Bax, activate caspase 3, and then cause cell death or apoptosis [37]. Linkermann et al. [38] pointed out that calpain was involved in programmed cell necrosis in acute kidney injury. Yamashima et al. [39] put forward the hypothesis of "calpain-cathepsin." Ischemia and hypoxia activate calpain that, in turn, acts on lysosomes, changing or disrupting lysosomal membrane permeability, leading to cathepsin in lysosomes being released into the cytoplasm. Recent studies have shown that the physical interaction between TRPC6 and podocyte calpain regulates podocyte cytoskeleton, cell adhesion, and movement [20].

Lysosomes are involved in cell processes such as secretion, cell signal transduction, and energy metabolism to maintain cell stability [40]. The kidney is the organ with the highest lysosome content; the integration of lysosome membrane protein and soluble lysosome hydrolase mediates lysosome activity. New evidence shows that lysosome protease cathepsin (CTSS) plays an essential role in the pathogenesis and progress of kidney diseases $[19,41]$. The main physiological function of CTSS is beyond lysosomes, and it participates in the degradation of extracellular matrix proteins [19].

The western blot validation experiment in this study suggested that CTSS elevated in the kidney of IMN patients. At the same time, Jobs et al. [42] found that the high circulating level of CTSS was related to the increased risk of human death due to CTSS involving complex pathways leading to impaired renal function. In addition, the upregulation of CTSS was detected in ochratoxin A-induced nephropathy [43], indicating that the findings of this study agree with previous work, suggesting that the high CTSS expression in IMN may be of great significance. It has also been found that, in mice, serum CTSS level and markers of inflammation-related endothelial dysfunction, such as soluble tumor necrosis factor receptor (sTNFR1, sTNFR12), increase with the decrease of eGFR. By contrast, in humans, eGFR increase is related to a reduction of CTSS [44]. These findings indicate that CTSS activity increases with CKD progression, thus representing a potential marker of disease progression.
Matrix metalloproteinases (MMPs) can hydrolyze extracellular matrix (ECM) and cell adhesion molecules [21], which play a key role in renal fibrosis. Increasing evidence shows MMP imbalance in acute kidney injury, diabetic nephropathy, and glomerulonephritis [45].

The main roles of MMP-2 and MMP-9 are degrading collagen type IV [46], inducing the loss of cell connection, and promoting epithelial-mesenchymal transition (EMT) of tubular cells, which leads to tubular atrophy and fibrosis [45]. MMP14 light in activate MMP-2 and MMP-9 [47] and has non-proteolytic function. These molecules participate in all stages of the inflammatory process and renal fibrosis, eventually leading to the gradual decline of renal function [46, 48]. IMN is often accompanied by renal fibrosis, and we found that the expression of MMP14 increased significantly in IMN, but no obvious changes were found in MMP-2 and MMP-9. Some studies confirmed that the activity and abundance of MMP-2 and MMP-9 decreased in the early stage of diabetic nephropathy; therefore, renal function damage may be related to the decrease in MMP-2 and MMP-9 activities.

The protease network is very complex, and some factors may hinder the relationship between natural peptides and kidney damage. The relationship between endogenous peptides and proteases should be carefully explained based on experiments. This study revealed that the dysregulation of urinary peptides in IMN might be related to proteases, providing clues for understanding the basic mechanism of IMN and exploring the therapeutic targets. However, this study still has some shortcomings: First, pooled samples were used in the study. Because the production of urine protein is affected by pre-kidney, the kidney itself, and post-kidney factors, interindividual differences will interfere with experimental results. Second, the amino acid sequence of a peptide has not been identified, which cannot explain the role of a certain peptide and its corresponding protease in diseases. Third, the mRNA and protein expression levels of proteases are not completely consistent. Therefore, the correlation analysis between the 24-h urine protein quantification, serum creatinine, cystatin $\mathrm{C}$, and the expression level of proteases may explain the significance of proteases in kidney function injury. The lack of this analysis is also a deficiency, which shall be addressed in future studies.

\section{Conclusions}

Our findings provide valuable insights to deepen our understanding of the role of proteases in IMN and highlight the potential significance of calpain, cathepsin, and MMPs in the pathogenesis of this disease.

\section{Methods}

\section{Subjects}

Random urine samples for peptidomics were collected from patients with biopsy-proven IMN $(n=22)$, minimal 
change disease (MCN, $n=5$ ), focal segmental glomerulosclerosis (FSGS, $n=5$ ), and IgA nephropathy (IgAN, $n$ $=5$ ), treated at the Shengjing Hospital of China Medical University from February 2019 to October 2019. Random urine samples from healthy controls $(\mathrm{HC}, n=20)$ were simultaneously collected. All patients were aged 18-75 years; the sex and age of the HCs matched those of the case group; HCs met the criteria for normal blood routine, urine routine, and kidney and liver function tests. Patients with kidney disease secondary to other diseases, autoimmune diseases, endocrine system diseases, tumors, and pregnancy complicated with kidney diseases and those receiving immunosuppressant or hormone therapy were excluded. Urine samples were divided into three groups: HC, IMN, and other kidney disease (OD, including MCN, IgAN, and FSGS).

Kidney tissue samples of patients with biopsy-proven IMN $(n=3)$ and adjacent non-cancerous tissues (NATs, $n=3$ ) from the biological sample bank of Shengjing Hospital of China Medical University were used for validation experiments. IMN samples were sourced from patients who met the above inclusion and exclusion criteria. This study was approved by the Ethics Committee of Shengjing Hospital of China Medical University (No. 2019PS194K); the requirement for informed consent of the patients was waived by this committee. All methods were carried out in accordance with relevant guidelines and regulations.

\section{Urine collection and urinary peptide extraction and digestion}

Random urine samples were collected in the morning into $10 \mathrm{~mL}$ polypropylene centrifuge tubes, avoiding mixing with pollutants, and centrifuged at 1,500 rpm for $5 \mathrm{~min}$ at room temperature. The supernatant was stored at $-80{ }^{\circ} \mathrm{C}$. Next, $200 \mu \mathrm{L}$ of urine sample was concentrated, and $10 \mu \mathrm{L}$ of pure water was added to it to dissolve the protein. Peptides were extracted using weak cation magnetic beads (WC-MB) according to the manufacturer's instructions (Yixinbochuang, Beijing, China). Extracted peptides were stored at $-80{ }^{\circ} \mathrm{C}$.

Urinary peptides extracted from HC, IMN, and OD groups were pooled into group-specific samples and then analyzed via a bottom-up proteomics strategy. The identified proteins were termed peptide-matched proteins. Dithiothreitol (Sigma, Oakville, Canada) was added to the sample to obtain a final concentration of $5 \mathrm{mM}$ and then reduced at $56{ }^{\circ} \mathrm{C}$ for $30 \mathrm{~min}$. Iodoacetamide (VETEC, Shanghai, China) was added to a final concentration of $11 \mathrm{mM}$ and incubated for $15 \mathrm{~min}$ at room temperature in the dark. Trypsin (Promega V5111, Madison, WI) was added at a ratio of 1:50 (protease: protein, $\mathrm{m} / \mathrm{m}$ ) and incubated overnight at $37{ }^{\circ} \mathrm{C}$. Then trypsin was added at a ratio of 1:100 (protease: protein, $\mathrm{m} / \mathrm{m}$ ) and incubated for a further $4 \mathrm{~h}$. The salts were then removed from peptides using Strata $X$ (Phenomenex, USA), and the peptides were redissolved in TEAB/ water (1:1 v/v) (SIGMA, Buchs, Switzerland).

\section{Matrix-assisted laser desorption/ionization time-of-flight MS (MALDI-TOF-MS)}

Peptide fingerprint of each sample (not pooled) was profiled using MALDI-TOF-MS on a Clin-TOF-II instrument (Clin-TOF-II, Yixinbochuang, China). First, $1 \mu \mathrm{L}$ of extracted peptide eluate was spotted onto the AnchorChip target and allowed to air dry. Next, $1 \mu \mathrm{L}$ of matrix solution containing $\alpha$-cyano-4-hydro-xycinnamic acid (Yixinbochuang, China) was spotted onto the same spot and air-dried and ionized using a nitrogen laser $(\lambda=337 \mathrm{~nm})$ operating at $40 \mathrm{~Hz}$. After running the MALDI-TOF-MS, mass calibration was performed. For each MALDI spot, 500 spectra were acquired (100 laser shots at five different spot positions) and processed using BioExplorer software.

\section{DIA-MS}

Peptides were dissolved in mobile phase A for liquid chromatography and separated with a NanoElute ultrahigh-performance liquid chromatography system (Germany, Bruker). Mobile phase A was an aqueous solution containing $0.1 \%$ formic acid and $2 \%$ acetonitrile. Mobile phase B was a solution containing $0.1 \%$ formic acid and $100 \%$ acetonitrile. Liquid-phase gradient settings were as follows: $0-70 \mathrm{~min}, 6-24 \% \mathrm{~B} ; 70-84 \mathrm{~min}$, $24-35 \%$ B; $84-87$ min, $35-80 \%$ B; $87-90$ min, $80 \%$ B; flow rate maintained at $450 \mathrm{~nL} / \mathrm{min}$. The peptide was separated using an ultra-high-performance liquid-phase system, injected into a capillary ion source for ionization, and then analyzed using MS (timsTOF Pro, Bruker, Germany). The ion source voltage was set at $2.0 \mathrm{kV}$, and the parent ions of peptide segments and their secondary fragments were detected and analyzed by highresolution TOF. The scanning range of secondary MS was set to 100-1700. Parallel accumulation serial fragmentation (PASEF) mode was used in the data acquisition mode. A secondary spectrogram with the charge number of parent ions in the range of $0-5$ was collected 10 times in the PASEF mode after collection of the primary mass spectrum, and the dynamic exclusion time of tandem MS scanning was set to $30 \mathrm{~s}$ to avoid repeated scanning of parent ions.

\section{Data analysis}

Raw data were searched against Maxquant 1.6.6.0. The setting of retrieval parameters was as follows. The database was Homo_sapiens_9606(20,366 sequences), and an anti-database was added to calculate the false discovery rate (FDR) caused by random matching. The 
quantitative method was set to label-free quantification (LFQ), and the FDR of protein identification and peptide-spectrum match (PSM) identification was set to $1 \%$. The quantitative calculation method of protein was as follows. The signal abundance of proteins in each sample was detected using MS. The LFQ intensity of proteins in each sample was obtained using the LFQ calculation method. The ratio of protein LFQ intensity between two different samples was considered the differential expression ratio for the comparison group.

\section{Bioinformatics analysis}

Gene Ontology (GO) and Kyoto Encyclopedia of Genes and Genomes (KEGG) signaling pathway enrichment analyses of proteins were carried out using the DAVID 6.8 online tool [49-52] (https://david.ncifcrf.gov/home. jsp). The interaction network of biological processes was analyzed and visualized using Cytoscape 3.8.1 [53], ClueGO 2.5.7 [54] software, Wukong cloud online platform [55], and GraphPad Prism 8.0 software.

\section{Protease prediction}

Proteasix [56] is an online open-source protease prediction tool focused on peptides and is specially used to predict the cleavage site of proteins and the corresponding proteases. In brief, Proteasix uses the information about natural peptides, UniProt ID, and the starting and ending amino acid positions of corresponding proteins to predict potential proteases. Proteasix retrieves information about cleavage sites from protease databases (MEROPS, BRENDA) and considers restriction of cleavage sites (from ENZYME database). Most human proteases have several cleavage sites, i.e., one peptide sequence can be cut by different proteases.

\section{Validation based on database analysis Analysis of tissue source of urinary peptides}

The open-source sc-RNA seq database Single Cell Portal contains a single-cell sequencing dataset. SCP211 dataset [57] containing single-cell sequencing data of normal adult kidneys was selected to analyze the tissue source of urinary peptide-matched proteins related to IMN. The cell types involved in this dataset are podocytes, endothelial cells, pericytes, proximal convoluted tubular cells (PCTs), proximal straight tubular cells (PSTs), thin descending limb (TDL), thin ascending limb (TAL), collecting duct-principal cells (CD-PCs), collecting duct-Aintercalated cells, CD-A-ICs), distal convoluted tubule (DCT), mesangial cells (Mes), fibroblasts (Fib), venous smooth muscle cells (vSMC), macrophages, natural killer cells (NKs), and T cells. If the protein was not expressed in the above kidney cells, it was considered that the protein in the urine was not from the kidney.
Validation of differentially expressed proteins in urine and kidney tissues of patients with IMN

iProX [58] is an open-source integrated proteomics resource; the dataset IPX0002813000 was produced from our laboratory under the scope of another project whose results have not yet been published. This dataset contains data from kidney tissues of IMN patients and normal controls. We analyzed the expression levels of urinary peptide-matched proteins in the kidney tissues based on this dataset.

\section{Validation of protease expression in the glomerulus}

The preprocessed gene expression data set GSE 99,339 [59] was downloaded from the Gene Expression Omnibus (GEO) database (https://www.ncbi.nlm.nih.gov/ $\mathrm{GEO} /$ ). This dataset contains mRNA expression data of kidney glomeruli obtained using laser microdissection from patients with FSGS $(n=12)$, IgAN $(n=26), \mathrm{MCN}$ $(n=8)$, and IMN $(n=21)$ and HCs $(n=8)$. The expression patterns of protease genes in different kidney diseases were examined.

\section{Western blot analysis}

To extract the proteins from the tissues, 3 IMN kidney tissues and 3 NATs were cut into pieces and lysed on ice using RIPA lysis buffer (Applygen, China) supplemented with Protease Inhibitor Cocktail, and then quantified using the BCA Protein Assay Kit. According to the expression of the internal reference protein GAPDH in the specimen, $90 \mu \mathrm{g}$ extracted proteins from IMN kidney tissue and $10 \mu \mathrm{g}$ extracted proteins NATs were separated by $12 \%$ SDS-PAGE and transferred to a nitrocellulose (NC) membrane (Millipore, Darmstadt, Germany). After blocking with $5 \%$ non-fat dry milk in tris-buffered saline and $0.1 \%$ Tween 20 solution, membranes were incubated overnight at $4{ }^{\circ} \mathrm{C}$ with primary antibodies against the following proteins: MMP9 (ABGENT, China, 1:1000), CAPN1 (Abclonal, China, 1:1000), MMP9, MMP14, CTSS, and GAPDH (Proteintech, China, 1: 5000). The membranes were then incubated with the appropriate HRP-Goat Anti-Rabbit (Elabscience, China, 1: 2,500) and HRP-Sheep Anti-Mouse (Jackson ImmunoResearch, USA, 1:8,000) secondary antibody for $1 \mathrm{~h}$ at room temperature. The specific bands were detected using an ECL detection kit (Applygen, China) and captured on an ImageQuant LAS 4000 mini system (GE Healthcare, NJ, USA). Relative expression was determined by normalizing to GAPDH expression using the ImageJ software (Version 1.51j, National Institutes of Health, MD, USA).

\section{Statistical analysis}

GraphPad Prism 8.0 was used for statistical analysis. Chi-square test was used to compare gender 
composition among groups; one-way analysis of variance (ANOVA) was used to compare differences between groups for quantitative indicators. Data are presented as mean \pm standard deviation. $P<0.05$ was considered statistically significant.

\section{Abbreviations}

IMN: Idiopathic membranous nephropathy; DIA-MS: Data-independent acquisition mass spectrometry; GEO: Gene Expression Omnibus; PLA R: Phospholipase A $A_{2}$ receptor; CKD: Chronic kidney disease; sc-RNA seq: Single-cell RNA sequencing; MCN: Minimal change disease; FSGS: Focal segmental glomerulosclerosis; IgAN: IgA nephropathy; NATs: Non-cancerous adjacent tissues; KDIGO: Kidney Disease Improving Global Outcomes; Cr: Creatinine; CysC: Cystatin C; eGFR: Estimated glomerular filtration rate; CAPN1: Calpain 1; CTSS: Cathepsin S; MMP: Matrix metalloproteinase

\section{Supplementary information}

The online version contains supplementary material available at https://doi. org/10.1186/s12864-021-08155-3.

\section{Additional file 1 \\ Additional file 2 \\ Additional file 3 \\ Additional file 4 \\ Additional file 5}

\section{Acknowledgements}

We gratefully acknowledge the reviewers for their comments and suggestions.

\section{Authors' contributions}

Lin B collected the urine samples, performed the experiments, wrote the main manuscript, and prepared figures. Chen $S$ and $W u Y$ collected the plasma samples and clinical information. Liu J, Bai Y, Liu Q, Zhang Y, and Qin $X$ guided the experiments, supervised the work, edited the manuscript, and reviewed the data during revision. All authors read and approved the final manuscript.

\section{Funding}

This work was supported by Liaoning Provincial Natural Science Foundation Project (NO. 20180550523), Liaoning Province Central Government's special project to guide local scientific and technological development (NO. 2019JH6/10400009), Major Special Project of Construction Program of China Medical University in 2018 (NO. 112/3110118034), Guangdong Province Major key projects of industrial technology (NO. 201902010003), and The 345 Talent Project of Shengjing Hospital of China Medical University (NO. 50 A).

\section{Availability of data and materials}

All data generated or analyzed in this study are included in this manuscript and its supplementary information files. Gene expression profiles GSE 99,339 was available from the Gene Expression Omnibus (GEO) database (https:// www.ncbi.nlm.nih.gov/GEO/).

\section{Declarations}

\section{Ethics approval and consent to participate}

This study was approved by the Medical Ethics Committee of Shengjing Hospital of China Medical University (No.2019PS194K); the requirement for informed consent of patients was waived by this committee. All methods were carried out in accordance with relevant guidelines and regulations.

\section{Consent for publication}

Not applicable.

\section{Competing interests}

The authors declare no competing interests.
Received: 5 April 2021 Accepted: 25 October 2021

Published online: 24 November 2021

\section{References}

1. Couser W G. Primary Membranous Nephropathy. Clin J Am Soc Nephrol. 2017;12:983-97.

2. Rovin BH, Caster DJ, Cattran DC, Gibson KL, Hogan J, Moeller MJ, Roccatello D, Cheung M, Wheeler DC, Winkelmayer WC, Floege J; Conference Participants. Management and treatment of glomerular diseases (part 2): conclusions from a Kidney Disease: Improving Global Outcomes (KDIGO) Controversies Conference. Kidney Int. 2019:95:281-95.

3. Kopp JB, Anders HJ, Susztak K, Podestà MA, Remuzzi G, Hildebrandt F, Romagnani P. Podocytopathies. Nat Rev Dis Primers. 2020;6:68.

4. Keri KC, Blumenthal S, Kulkarni V, Beck L, Chongkrairatanakul T. Primary membranous nephropathy: comprehensive review and historical perspective. Postgrad Med J. 2019;95:23-1.

5. Thokhonelidze I, Maglakelidze N, Sarishvili N, Kasradze T, Dalakishvili K. Association of anti-phospholipasea2-receptor antibodies with clinical course of idiopathic membranous nephropathy. Georgian Med News. 2015;241:4953.

6. Sever S, Altintas MM, Nankoe SR, Möller CC, Ko D, Wei C, Henderson J, del Re EC, Hsing L, Erickson A, Cohen CD, Kretzler M, Kerjaschki D, Rudensky A, Nikolic B, Reiser J. Proteolytic processing of dynamin by cytoplasmic cathepsin L is a mechanism for proteinuric kidney disease. J Clin Invest. 2007;117:2095-104.

7. Madhusudhan T, Kerlin BA, Isermann B. The emerging role of coagulation proteases in kidney disease. Nat Rev Nephrol. 2016;12:94-109.

8. Vandenbroucke RE, Libert C. Is there new hope for therapeutic matrix metalloproteinase inhibition?. Nat Rev Drug Discov. 2014;13:904-927.

9. Altintas MM, Moriwaki K, Wei C, Möller CC, Flesche J, Li J, Yaddanapudi S, Faridi MH, Gödel M, Huber TB, Preston RA, Jiang JX, Kerjaschki D, Sever S, Reiser J. Reduction of proteinuria through podocyte alkalinization. J Biol Chem. 2014:289:17454-67.

10. Bauça JM, Martínez-Morillo E, Diamandis EP. Peptidomics of urine and other biofluids for cancer diagnostics. Clin Chem. 2014;60:1052-61.

11. Van JAD, Scholey JW, Konvalinka A. Insights into Diabetic Kidney Disease Using Urinary Proteomics and Bioinformatics. J Am Soc Nephrol. 2017;28: 1050-61.

12. Santucci L, Bruschi M, Candiano G, Lugani F, Petretto A, Bonanni A, Ghiggeri GM. Urine Proteome Biomarkers in Kidney Diseases. I. Limits, Perspectives, and First Focus on Normal Urine. Biomark Insights. 2016:11:41-8.

13. Karczewski KJ, Snyder MP. Integrative omics for health and disease. Nat Rev Genet. 2018;19:299-310.

14. Graterol F, Navarro-Muñoz M, Ibernon M, López D, Troya Ml, Pérez V, Bonet J, Romero R. Poor histological lesions in IgA nephropathy may be reflected in blood and urine peptide profiling. BMC Nephrol. 2013;14:82.

15. Krochmal M, Kontostathi $G$, Magalhães $P$, Makridakis M, Klein J, Husi H, Leierer J, Mayer G, Bascands JL, Denis C, Zoidakis J, Zürbig P, Delles C, Schanstra JP, Mischak H, Vlahou A. Urinary peptidomics analysis reveals proteases involved in diabetic nephropathy. Sci Rep. 2017;7:15160.

16. Schanstra JP, Zürbig P, Alkhalaf A, Argiles A, Bakker SJ, Beige J, Bilo HJ, Chatzikyrkou C, Dakna M, Dawson J, Delles C, Haller H, Haubitz M, Husi H, Jankowski J, Jerums G, Kleefstra N, Kuznetsova T, Maahs DM, Menne J, Mullen W, Ortiz A, Persson F, Rossing P, Ruggenenti P, Rychlik I, Serra AL, Siwy J, Snell-Bergeon J, Spasovski G, Staessen JA, Vlahou A, Mischak H, Vanholder R. Diagnosis and Prediction of CKD Progression by Assessment of Urinary Peptides. J Am Soc Nephrol. 2015;26:1999-2010.

17. Shimura T, Dayde D, Wang H, Okuda Y, Iwasaki H, Ebi M, Kitagawa M, Yamada T, Yamada T, Hanash SM, Taguchi A, Kataoka H. Novel urinary protein biomarker panel for early diagnosis of gastric cancer. $\mathrm{Br} J$ Cancer. 2020:123:1656-1664

18. Virreira Winter S, Karayel O, Strauss MT, Padmanabhan S, Surface M, Merchant K, Alcalay RN, Mann M. Urinary proteome profiling for stratifying patients with familial Parkinson's disease. EMBO Mol Med. 2021;13:e13257.

19. Cocchiaro P, De Pasquale V, Della Morte R, Tafuri S, Avallone L, Pizard A, Moles A, Pavone LM. The Multifaceted Role of the Lysosomal Protease Cathepsins in Kidney Disease. Front Cell Dev Biol. 2017;5:114.

20. Farmer LK, Rollason R, Whitcomb DJ, Ni L, Goodliff A, Lay AC, Birnbaumer L, Heesom KJ, Xu SZ, Saleem MA Welsh Gl. TRPC6 Binds to and Activates Calpain, Independent of Its Channel Activity, and Regulates Podocyte 
Cytoskeleton, Cell Adhesion, and Motility. J Am Soc Nephrol. 2019;30:191024.

21. Zakiyanov O, Kalousová M, Zima T, Tesař V. Matrix Metalloproteinases in Renal Diseases: A Critical Appraisal. Kidney Blood Press Res. 2019;44:298-330

22. Siwy J, Zürbig P, Argiles A, Beige J, Haubitz M, Jankowski J, Julian BA, Linde PG, Marx D, Mischak H, Mullen W, Novak J, Ortiz A, Persson F, Pontillo C, Rossing P, Rupprecht H, Schanstra JP, Vlahou A, Vanholder R. Noninvasive diagnosis of chronic kidney diseases using urinary proteome analysis. Nephrol Dial Transplant. 2017;32:2079-2089.

23. Zhang M, Fu G, Lei T. Two urinary peptides associated closely with type 2 diabetes mellitus. PLoS One. 2015;10:e0122950.

24. Lazar C, Gatto L, Ferro M, Bruley C, Burger T. Accounting for the Multiple Natures of Missing Values in Label-Free Quantitative Proteomics Data Sets to Compare Imputation Strategies. J Proteome Res. 2016;15:1116-25.

25. Jing J, Gao Y. Urine biomarkers in the early stages of diseases: current status and perspective. Discov Med. 2018;25:57-65.

26. Ngai HH, Sit WH, Jiang PP, Xu RJ, Wan JM, Thongboonkerd V. Serial changes in urinary proteome profile of membranous nephropathy: implications for pathophysiology and biomarker discovery. J Proteome Res. 2006;5:3038-47.

27. Pang L, Li Q, Li Y, Liu Y, Duan N, Li H. Urine proteomics of primary membranous nephropathy using nanoscale liquid chromatography tandem mass spectrometry analysis. Clin Proteomics. 2018;15:5.

28. Brondani LA, Soares AA, Recamonde-Mendoza M, Dall'Agnol A, Camargo JL, Monteiro KM, Silveiro SP. Urinary peptidomics and bioinformatics for the detection of diabetic kidney disease. Sci Rep. 2020;10:1242.

29. Buccitelli C, Selbach M. mRNAs, proteins and the emerging principles of gene expression control. Nat Rev Genet. 2020;21:630-644.

30. Liu K, Lyu L, Chin D, Gao J, Sun X, Shang F, Caceres A, Chang ML, Rowan S, Peng J, Mathias R, Kasahara H, Jiang S, Taylor A. Altered ubiquitin causes perturbed calcium homeostasis, hyperactivation of calpain, dysregulated differentiation, and cataract. Proc Natl Acad Sci USA. 2015;112:1071-6.

31. Hanna RA, Campbell RL, Davies PL. Calcium-bound structure of calpain and its mechanism of inhibition by calpastatin. Nature. 2008;456:409-412.

32. Siklos M, BenAissa M, Thatcher GR. Cysteine proteases as therapeutic targets: does selectivity matter? A systematic review of calpain and cathepsin inhibitors. Acta Pharm Sin B. 2015;5:506-519.

33. Dryer SE, Roshanravan H, Kim EY. TRPC channels: Regulation, dysregulation and contributions to chronic kidney disease. Biochim Biophys Acta Mol Basis Dis. 2019;1865:1041-1066.

34. Salido GM, Sage SO, Rosado JA. TRPC channels and store-operated $\mathrm{Ca}(2+)$ entry. Biochim Biophys Acta. 2009;1793:223-30.

35. Hofstra JM, Coenen MJ, Schijvenaars MM, Berden JH, van der Vlag J, Hoefsloot LH, Knoers NV, Wetzels JF, Nijenhuis T. TRPC6 single nucleotide polymorphisms and progression of idiopathic membranous nephropathy. PLoS One. 2014;9:e102065.

36. Azuma M, Shearer TR. The role of calcium-activated protease calpain in experimental retinal pathology. Surv Ophthalmol. 2008;53:150-163.

37. Gao G, Dou QP. N-terminal cleavage of bax by calpain generates a potent proapoptotic $18-\mathrm{kDa}$ fragment that promotes bcl-2-independent cytochrome C release and apoptotic cell death. J Cell Biochem. 2000;80:5372.

38. Linkermann A, De Zen F, Weinberg J, Kunzendorf U, Krautwald S. Programmed necrosis in acute kidney injury. Nephrol Dial Transplant. 2012; 27:3412-3419.

39. Yamashima T, Kohda Y, Tsuchiya K, Ueno T, Yamashita J, Yoshioka T, Kominami E. Inhibition of ischaemic hippocampal neuronal death in primates with cathepsin B inhibitor CA-074: a novel strategy for neuroprotection based on 'calpain-cathepsin hypothesis'. Eur I Neurosci. 1998;10:1723-33.

40. Settembre C, Fraldi A, Medina DL, Ballabio A. Signals from the lysosome: a control centre for cellular clearance and energy metabolism. Nat Rev Mol Cell Biol. 2013;14:283-296.

41. Van JAD, Clotet-Freixas S, Hauschild AC, Batruch I, Jurisica I, Elia Y, Mahmud FH, Sochett E, Diamandis EP, Scholey JW, Konvalinka A. Urinary proteomics links keratan sulfate degradation and lysosomal enzymes to early type 1 diabetes. PLoS One. 2020;15:e0233639.

42. Jobs E, Ingelsson E, Risérus U, Nerpin E, Jobs M, Sundström J, Basu S, Larsson A, Lind L, Ärnlöv J. Association between serum cathepsin S and mortality in older adults. JAMA. 2011;306:1113-21.

43. Luhe A, Hildebrand H, Bach U, Dingermann T, Ahr HJ. A new approach to studying ochratoxin A (OTA)-induced nephrotoxicity: expression profiling in vivo and in vitro employing cDNA microarrays. Toxicol Sci. 2003;73:31528.

44. Steubl D, Kumar SV, Tato M, Mulay SR, Larsson A, Lind L, Risérus U, Renders L, Heemann U, Carlsson AC, Ärnlöv J, Anders HJ. Circulating cathepsin-S levels correlate with GFR decline and sTNFR1 and sTNFR2 levels in mice and humans. Sci Rep. 2017;7:43538.

45. Tan RJ, Liu Y. Matrix metalloproteinases in kidney homeostasis and diseases. Am J Physiol Renal Physiol. 2012;302:F1351-F1361.

46. Hu X, Beeton C. Detection of functional matrix metalloproteinases by zymography. J Vis Exp. 2010;45:2445.

47. Cheng Z, Limbu MH, Wang Z, Liu J, Liu L, Zhang X, Chen P, Liu B. MMP-2 and 9 in Chronic Kidney Disease. Int J Mol Sci. 2017;18:776.

48. Provenzano M, Andreucci M, Garofalo C, Faga T, Michael A, lelapi N, Grande R, Sapienza P, Franciscis S, Mastroroberto P, Serra R. The Association of Matrix Metalloproteinases with Chronic Kidney Disease and Peripheral Vascular Disease: A Light at the End of the Tunnel? Biomolecules. 2020;10: 154.

49. Huang da W, Sherman BT, Lempicki RA. Bioinformatics enrichment tools: paths toward the comprehensive functional analysis of large gene lists. Nucleic Acids Res. 2009;37:1-13.

50. Kanehisa M, Goto S. KEGG: kyoto encyclopedia of genes and genomes. Nucleic Acids Res. 2000;28(1):27-30.

51. Kanehisa M. Toward understanding the origin and evolution of cellular organisms. Protein Sci. 2019;28(11):1947-1951.

52. Kanehisa M, Furumichi M, Sato Y, Ishiguro-Watanabe M, Tanabe M. KEGG: integrating viruses and cellular organisms. Nucleic Acids Res. 2021;49(D1): D545-D551.

53. Franz M, Lopes $C T$, Huck G, Dong Y, Sumer O, Bader GD. Cytoscape.js: a graph theory library for visualisation and analysis. Bioinformatics. 2016;32: 309-11.

54. Bindea G, Mlecnik B, Hackl H, Charoentong P, Tosolini M, Kirilovsky A, Fridman WH, Pagès F, Trajanoski Z, Galon J. ClueGO: a Cytoscape plug-in to decipher functionally grouped gene ontology and pathway annotation networks. Bioinformatics. 2009;25:1091-3.

55. Wang S, Zheng W, Hu L, Gong M, Yang H. MixProTool: A Powerful and Comprehensive Web Tool for Analyzing and Visualizing Multigroup Proteomics Data. J Comput Biol. 2018;25:1123-27.

56. Klein J, Eales J, Zürbig P, Vlahou A, Mischak H, Stevens R. Proteasix: a tool for automated and large-scale prediction of proteases involved in naturally occurring peptide generation. Proteomics. 2013;13:1077-82.

57. Subramanian $A$, Sidhom EH, Emani M, Vernon $K$, Sahakian $N$, Zhou Y, KostAlimova M, Slyper M, Waldman J, Dionne D, Nguyen LT, Weins A, Marshall $J$, Rosenblatt-Rosen O, Regev A, Greka A. Single cell census of human kidney organoids shows reproducibility and diminished off-target cells after transplantation. Nat Commun. 2019;10:5462.

58. Ma J, Chen T, Wu S, Yang C, Bai M, Shu K, Li K, Zhang G, Jin Z, He F, Hermjakob H, Zhu Y. iProX: an integrated proteome resource. Nucleic Acids Res. 2019:47:D1211-D1217.

59. Grayson PC, Eddy S, Taroni JN, Lightfoot YL, Mariani L, Parikh H, Lindenmeyer MT, Ju W, Greene CS, Godfrey B, Cohen CD, Krischer J, Kretzler M, Merkel PA; Vasculitis Clinical Research Consortium, the European Renal cDNA Bank cohort, and the Nephrotic Syndrome Study Network. Metabolic pathways and immunometabolism in rare kidney diseases. Ann Rheum Dis. 2018;77:1226-33.

\section{Publisher's Note}

Springer Nature remains neutral with regard to jurisdictional claims in published maps and institutional affiliations. 